\title{
C-reactive protein but not procalcitonin may predict antibiotic response and outcome in infections following major abdominal surgery
}

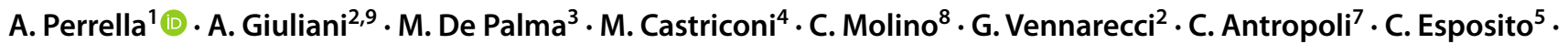 \\ F. Calise ${ }^{10} \cdot$ A. Frangiosa ${ }^{6} \cdot$ Infection in Surgery Study Group AORN A. Cardarelli
}

Received: 4 August 2021 / Accepted: 16 September 2021 / Published online: 26 October 2021

(C) Italian Society of Surgery (SIC) 2021

\begin{abstract}
We aimed to evaluate the usefulness of C-reactive protein (CRP) and procalcitonin (PCT) as markers of infection, sepsis and as predictors of antibiotic response after non-emergency major abdominal surgery. We enrolled, from June 2015 to June 2019, all patients who underwent surgery due to abdominal infection (peritoneal abscess, peritonitis) or having sepsis episode after surgical procedures (i.e. hepatectomy, bowel perforation, pancreaticoduodenectomy (PD), segmental resection of the duodenum (SRD) or biliary reconstruction in a Tertiary Care Hospital. Serum CRP (cut-off value $<5 \mathrm{mg} / \mathrm{L}$ ) and PCT (cut-off value $<0.1 \mathrm{mcg} / \mathrm{L}$ ) were measured in the day when fever was present or within $24 \mathrm{~h}$ after abdominal surgery. Both markers were assessed every $48 \mathrm{~h}$ to follow-up antibiotic response and disease evolution up to disease resolution. We enrolled a total of 260 patients underwent non-emergency major abdominal surgery and being infected or developing infection after surgical procedure with one or more microbes (55\% mixed Gram-negative infection including Klebsiella KPC, 35\% Gram-positive infection, $10 \%$ with Candida infection), 58\% of patients had ICU admission for at least $96 \mathrm{~h}, 42 \%$ of patients had fast track ICU (48 h). In our group of patients, we found that PCT had a trend to increase after surgical procedure; particularly, those undergoing liver surgery had higher PCT than those underwent different abdominal surgery ( $U$ Mann-Whitney $p<0.05$ ). CRP rapidly increase after surgery in those developing infection and showed a statistical significant decrease within $48 \mathrm{~h}$ in those subject being responsive to antibiotic treatment and having a clinical response within 10 days independently form the pathogens (bacterial or fungal). Further we found that those having CRP higher than $250 \mathrm{mg} / \mathrm{L}$ had a reduced percentage of success treatment at 10 days compared to those $<250 \mathrm{mg} / \mathrm{mL}$ ( $U$ Mann-Whitney $p<0.05$ ). PCT did not show any variation according to treatment response. CRP in our cohort seems to be a useful marker to predict antibiotic response in those undergoing non-emergency abdominal surgery, while PCT seem to be increased in those having major liver surgery, probably due to hepatic production of cytokines.
\end{abstract}

Keywords Infection · Antimicrobialstewardship · CRE - Abdominal surgery $\cdot$ Surgical infection · Inflammation · Procalcitonin $\cdot$ CRP

On behalf of Cardarelli Infectious Disease Study Group.

The members of Infection in Surgery Study Group AORN

A.Cardarelli are mentioned in "Acknowledgements" section.

A. Perrella

alessandro.perrella@aocardarelli.it

1 Infectious Disease Service at Health Direction Hospital A. Cardarelli, Naples, Italy

2 Hepatobiliary Surgery and Liver Transplant Center Hospital A. Cardarelli, Naples, Italy

3 General Surgery Hospital A. Cardarelli, Naples, Italy

4 Emergency Surgery Hospital A. Cardarelli, Naples, Italy
5 Liver Intensive Care Unit Hospital A. Cardarelli, Naples, Italy

6 Intensive Care Unit Hospital A. Cardarelli, Naples, Italy

7 III Surgical Unit Hospital A. Cardarelli, Naples, Italy

8 I Surgical Unit Hospital A. Cardarelli, Naples, Italy

9 Surgical Unit, San Carlo Hospital, Potenza, Italy

10 Surgical Unit Pineta Grande Hospital, Caserta, Italy 


\section{Introduction}

Infections following surgical procedures are one of the major cause of morbidity and mortality, particularly after abdominal surgery where they could evolve in a more critical conditions as sepsis [1]. Classification of these infections is based according to surgical site infections (SSIs) or distant infections such as, respiratory tract infections, urinary tract infections, catheter associated infections, as well as sepsis itself [2]. Several risk factors such as age, sex, body mass index (BMI), comorbidities like diabetes or organ failure, end-stage organ disease or previous oncological diseases requiring chemotherapy and/or radiotherapy, have been associated with a higher probability to develop the infections [3]. Coupled to these well-known risk factors should also be considered host factors related to surgical procedure, like operative time, blood transfusion, that may further predispose to hospital acquired infections too [3]. Therefore, markers to determine an earlier onset of infection and possible response to antibiotic are fundamental not only in terms of disease resolution but also in terms of antimicrobial stewardship strategy, minimizing the risk of antibiotic resistance [4]. At now, several papers have been published on the possible makers to manage infection after major abdominal surgery and two in particular seem to be relevant in clinical management of patients with infection after major abdominal surgery [5, 6]. C-reactive protein (CRP), is one of the first acute phase proteins to be described, being sensitive systemic marker of inflammation and tissue damage [7]. Procalcitonin (PCT), the prohormone of calcitonin, was first described as a biochemical marker of infection in 1993 [8]. Bacterial endotoxins are potent stimuli for PCT synthesis, which exhibits faster kinetics than CRP. PCT is released into the circulation 3-4 h after an injection of endotoxin, reaching peak levels after $8-24 \mathrm{~h}$, while CRP peaks at $36-50 \mathrm{~h}$ after stimulus [9]. This would make PCT more useful as an infection monitoring tool in the perioperative setting; however, discordant data are reported in abdominal sepsis and infection after major abdominal surgery [10, 11]. In this study, we aimed to evaluate the usefulness of C-reactive protein (CRP) and procalcitonin (PCT), in patients with infections and sepsis after major abdominal surgery, to early predict infection or surgical complications as well as the response to antibiotic treatment.

\section{Patients and methods}

\section{Study design}

We performed an observational study, from January 2015 to June 2019 , on 260 consecutive patients undergoing non-emergency major abdominal surgery, excluding liver transplantation, in a large tertiary care Hospital of South Italy (AORN A.Cardarelli) evaluating abdominal infections (peritoneal abscess, peritonitis) or having sepsis episode after major abdominal surgical procedures (i.e. hepatectomy, bowel perforation, pancreatoduodenectomy (PD) or segmental resection of the duodenum (SRD) or biliary reconstruction) and colorectal surgery. In these patients, we evaluated the trend of $\mathrm{C}$-reactive protein (CRP) and procalcitonin (PCT) and their possible correlation with postsurgical evolution, infection onset, anastomotic leak or dehiscence, antibiotic response as well as related ICU stay. The current study analysed a standard approach to infection according to diagnostic algorithm, infection source control, microbiological data, medical treatment and management of complications. All antibiotics were used according to their legal indications and license in Europe and Italy and Hospital and Regional guidelines and therefore no off-label empiric treatment were performed. However, no protocol specifications or interventions about the characteristics of patients, type of infection, antibiotic regimen and other treatments, or diagnostic measures and monitoring was managed except for laboratory markers above mentioned. The study was performed according to the ethical principles of the Declaration of Helsinki and of Good Clinical Practice and with applicable national regulatory and institutional standards. Complicated intrabdominal infections (cIAI) were diagnosed according to previous definition as stated by international guidelines [1,2]. Infections were diagnosed as nosocomial after $48 \mathrm{~h}$ (hrs) or more of hospitalization; communityacquired infections were defined as those diagnosed before this time point or for those patients admitted in hospital being already febrile [12]. Further, anamnestic evaluation for previous antibiotics use was analysed in all admitted patients. Serum CRP (cut-off value $<5 \mathrm{mg} / \mathrm{L}$ ) and PCT (cut-off value $<0.1 \mathrm{mcg} / \mathrm{L}$ ) were measured on the day of hospital admission according to centralized laboratory, at the following time points: day before surgical procedure (T0), $48 \mathrm{~h}$ after surgery (T1) and every $48 \mathrm{~h}$ as well as at the time of fever onset (T2,T3..Tn). Endotoxin activity assay (EAA) was also evaluated every $72 \mathrm{~h}$ in those having abdominal infections. All patients showing infection after surgery underwent to microbiological assessment and consequently empirically treated according to hospital and regional therapeutic protocol or infectious disease specialist schedule. Hospital treatment schedules in patients with critical condition after non-emergency abdominal surgery were as follows: Piperacillin/Tazobactam (4.5 gr every $8 \mathrm{~h}$ ) plus Metronidazole or Tigecycline (100 mg loading dose and thereafter $50 \mathrm{mg}$ every $12 \mathrm{~h}$ ) plus Piperacillin/ Tazobactam until June 2016. Beyond that date we had the following empirical or antibiogram based antibiotic schedule: Ceftolozano/Tazobactam $(1.5 \mathrm{gr}$ every $8 \mathrm{~h}$ ) plus 
Metronidazole (500 mg every $8 \mathrm{~h}$ ) or Tigecycline (100 mg loading dose and thereafter $50 \mathrm{mg}$ every $12 \mathrm{~h}$ ), or else Ceftolozano/Tazobactam plus Tigecylcine plus Fosfomycin (4 gr every $6 \mathrm{~h}$ ), or Ceftazidine/Avibactam plus Tigecycline. All treatment were used in patients with documented infection after major abdominal surgery requiring intensive care unit approach and/or with evidence of higher risk factors based on our previous experiences [13, 14]. Targeted antimicrobial treatment schedule was based according to microbiological culture isolates and MIC based on EUCAST; in case of Klebsiella KPC Infection a combined three antibiotic based regimen was managed with Meropenem plus Tigecycline plus Colistin or Fosfomycin when available. A carbapenem sparing policy was introduced starting from the end of June 2016; therefore, this class of antibiotics was limited in their use and replaced by Ceftolozane/Tazobactam or Ceftazidime/Avibactam in critical clinical condition or in patients admitted as second cure from other Hospitals. Carbapenems were used in any case only in condition were no other option were available according to regional guidelines and literature evidences. Response to treatment schedule was based on the following variables: resolution of fever, leukocyte count progressive reduction over $72 \mathrm{~h}$, weaning of inotropes drugs in those with sepsis and being in ICU [15] and the evaluation of CRP and PCT trend. Treatment failure was considered when patients had relapsing fever after 5 days treatment. Follow-up of antibiotic response and disease evolution was managed up 28th-day postsurgical procedure or up fever resolution as in-patient or out-patient setting according to clinical condition. Statistical analysis has been performed by Mann-Whitney U test or Kruskal-Wallis test to compare continuous variables and statistical differences in all evaluated subgroups (anastomosis dehiscence vs non anastomosis dehiscence or Antibiotic schedule up 2016 vs antibiotic schedule from 2016 up today). Univariate and Multivariate analyses were also performed. $\mathrm{P}$ values below 0.05 were considered statistically significant. All analyses were performed with the Graph Pad software (Mac Os 10.13), PSPP MacOs Bundles. Data are shown as either median or range, in the case of continuous variables or number and percentage, for categorical variable.

\section{Results}

In this study, we enrolled 260 consecutive patients underwent non-emergency major abdominal surgery (Table 1); 140 out 260 were classified as non-infection group (Group A) while 120 were found to be affected by infections at the time of surgery or after surgical procedure with one or more microbes (Group B) (Table 2). As first consideration we did not find any difference in age, sex, smoke, alcohol abuse,
Table 1 Baseline characteristics of the entire cohort of study $(N=260)$

\begin{tabular}{|c|c|}
\hline \multicolumn{2}{|l|}{ Parameter } \\
\hline Age (yrs), median [IQR] & $66.5[55.2-73.7]$ \\
\hline \multicolumn{2}{|l|}{ Sex, $n(\%)$} \\
\hline Male & $161(62)$ \\
\hline Female & 99 (38) \\
\hline BMI, median [IQR] & $25.5[22-25]$ \\
\hline Smoke, $(\%)$ & 25 \\
\hline Potus, $(\%)$ & 12 \\
\hline Diabetes, $n(\%)$ & 14 \\
\hline Metabolic syndrome, $n(\%)$ & 3 \\
\hline Lactates at EAB, median [IQR] & $1.15[0.73-1.15]$ \\
\hline \multicolumn{2}{|l|}{ ASA Score (as number) } \\
\hline ASA I & 13 \\
\hline ASA II & 127 \\
\hline ASA III & 40 \\
\hline \multicolumn{2}{|l|}{ Type of surgery (as number) } \\
\hline Cholecistectomy & 50 \\
\hline Hepatectomy & 80 \\
\hline CDP & 45 \\
\hline Segmental resection of the duodenum & 20 \\
\hline Total gastrectomy & 25 \\
\hline Partial gastrectomy & 16 \\
\hline Colon surgery ${ }^{\mathrm{a}}$ & 22 \\
\hline Abdominal abscess & 27 \\
\hline Fungal infections, $n(\%)$ & $20(7.6)$ \\
\hline \multicolumn{2}{|l|}{ Bacteria, $n(\%)$} \\
\hline Gram negative & $78(65)$ \\
\hline Gram positive & $42(35)$ \\
\hline
\end{tabular}

In table, all analyzed parameters are reported on overall patient population. Data are expressed ad either number and percentage or median and interquartile range (IQR)

$C D P$ cephalic duodeno-pancreatectomy

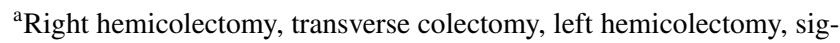
moidectomy

ASA, type of surgery and BMI distribution of enrolled patients in both groups. Among those in Group B we had 21 out 120 patients with postsurgical pneumonia, 15 out 120 patients had urinary tract infections and 84 out 120 patients had cIAI including abdominal abscess at the time of hospital admission. 12 out 21 patients with pneumonia had bacterial isolation during ICU stay being positive for not multidrug resistant Pseudomonas aeruginosa and Klebsiella spp. No microbiological data were available for 9 out 21 patients with hospital acquired pneumonia. 10 out 15 patients with urinary tract infections were positive to Klebsiella pneumoniae while the remaining was positive to Acinetobacter baumannii MDR. Eighty-four patients with cIAI, had a 65\% mixed Gram negative isolates including Klebsiella KPC ( $4.5 \%$ of total), Enterobacter spp (20\% with $15 \%$ of 
Table 2 Baseline characteristics according to infection: univariate and multivariate analysis $(n=260)$

\begin{tabular}{|c|c|c|c|}
\hline \multirow[t]{2}{*}{ Parameter } & \multicolumn{3}{|l|}{ Univariate analysis } \\
\hline & $\begin{array}{l}\text { Patients without infection } \\
\text { Group A }\end{array}$ & $\begin{array}{l}\text { Patients with infection } \\
\text { Group B }\end{array}$ & $p$ \\
\hline Number of Pts & 140 & 120 & \\
\hline Age (yrs), median [IQR] & $58.5[55.2-67]$ & $57.5[47-61.7]$ & 0.132 \\
\hline Sex, $n(\%)$ & & & 0.210 \\
\hline Male & $105(75)$ & $78(65)$ & \\
\hline Female & $35(25)$ & $42(35)$ & \\
\hline BMI, median [IQR] & $23[22-25]$ & $24[23-26]$ & 0.204 \\
\hline Smoke, $n(\%)$ & $20(14)$ & $8(10)$ & 0.364 \\
\hline Potus, $n(\%)$ & $7(5)$ & $8(5)$ & 0.329 \\
\hline Diabetes, $n(\%)$ & $3(12.5)$ & $5(25)$ & 0.436 \\
\hline Metabolic syndrome, $n(\%)$ & $2(8.3)$ & $5(25)$ & 0.217 \\
\hline SOFA Score, median [IQR] & $8[7-9]$ & $8[7,8]$ & 0.360 \\
\hline Lactates at $\mathrm{EAB}^{\mathrm{b}}$, median [IQR] & $2.3[1.85-3]$ & $2[1.55-3.75]$ & 0.849 \\
\hline \multicolumn{4}{|l|}{ Leukocytosis, median [IQR] } \\
\hline Baseline & $8.5[5.2-9.3]$ & $11[19-22.8]$ & 0.293 \\
\hline $72 \mathrm{~h}$ & $10.5[7-11.4]$ & $18[16-20]$ & 0.785 \\
\hline $96 \mathrm{~h}$ & $7.3[6.2-8.4]$ & 16 [15.2-18] & 0.414 \\
\hline \multicolumn{4}{|l|}{ PCT, median [IQR] } \\
\hline Baseline & $4.5[2-7.5]$ & $5.6[3.13-7.75]$ & 0.129 \\
\hline $48 \mathrm{~h}$ & $5.4[2-8]$ & $5.7[4-7.5]$ & 0.403 \\
\hline \multicolumn{4}{|l|}{ EAA, median [IQR] } \\
\hline Baseline & $0.4[0.5-0.6]$ & $0.65[0.4-0.7]$ & 0.457 \\
\hline $72 \mathrm{~h}$ & $0.56[0.5-0.6]$ & $0.68[0.5-0.7]$ & 0.772 \\
\hline \multicolumn{4}{|l|}{ PCR, median [IQR] } \\
\hline Baseline & $105[89-132.2]$ & $284.5[145.2-346.2]$ & 0.548 \\
\hline $48 \mathrm{~h}$ & 123 [108.2-174.7] & $126[110-197.2]$ & 0.03 \\
\hline ICU stay over 48 h, $n(\%)$ & $22(15.7)$ & $45(37.5)$ & 0.001 \\
\hline Abx response at $48 \mathrm{~h}, \mathrm{n}(\%)$ & $6(25)$ & $17(85)$ & 0.000 \\
\hline Exitus, $n(\%)$ & $15(12)$ & $10(8)$ & 1.000 \\
\hline Fungal infections, $n(\%)$ & $2(8.3)$ & $2(10)$ & 1.000 \\
\hline \multicolumn{4}{|l|}{ Bacteria, $n(\%)$} \\
\hline Gram negative & 0 & $78(65)$ & n.a \\
\hline $\mathrm{MDR}^{\mathrm{a}}$ & 0 & $16(13.3)$ & n.a \\
\hline Gram positive & 0 & $42(35)$ & n.a \\
\hline
\end{tabular}

Table shows the results related to the analysed variables in both groups. Statistically significant differences were found in ICU stay over 14 days and response at $48 \mathrm{~h}$. No differences have been found in bacterial infections

${ }^{a}$ Gram-negative MDR including Klebsiella KPC, in this case the following therapeutic schedule was used (Colimicine plus Tigecycline plus Meropenem

${ }^{b}$ Parameters after surgery. Data are expressed ad either number and percentage or median and interquartile range (IQR)
ESBL), E.coli (25\% with 35\% of ESBL), Acinetobacter baumanni (3.3\%), Klebsiella spp (22\%), Pseudomonas aeruginosa $15 \%$, other Gram negative (10.2\%) (Tables 1 and 2 ). On the other hand, $35 \%$ of isolated were Gram positive including Enterococcus spp non VRE (67\% of the isolates) or Staphylococcus (33\%) of whom MSSA (78\%) and MRSA (22\%). Fungal infections represented $7.6 \%$ of overall infections and were based on Candida albicans (75\%), Candida tropicalis (12\%), Candida Glabrata (8\%), Candida spp (5\%). $58 \%$ of patients had ICU admission for at least $72 \mathrm{~h}$ after surgical procedure, $42 \%$ of patients had fast track ICU, defined as less than $48 \mathrm{~h}$ intensive care stay. Our findings showed that PCT serum levels did not show any statistical significant difference among patients with or without infection after major abdominal surgery in all observed time points ( $U$ Mann-Whitney $p>0.05$ ). Indeed procalcitonin 


\section{CRP vs PCT}

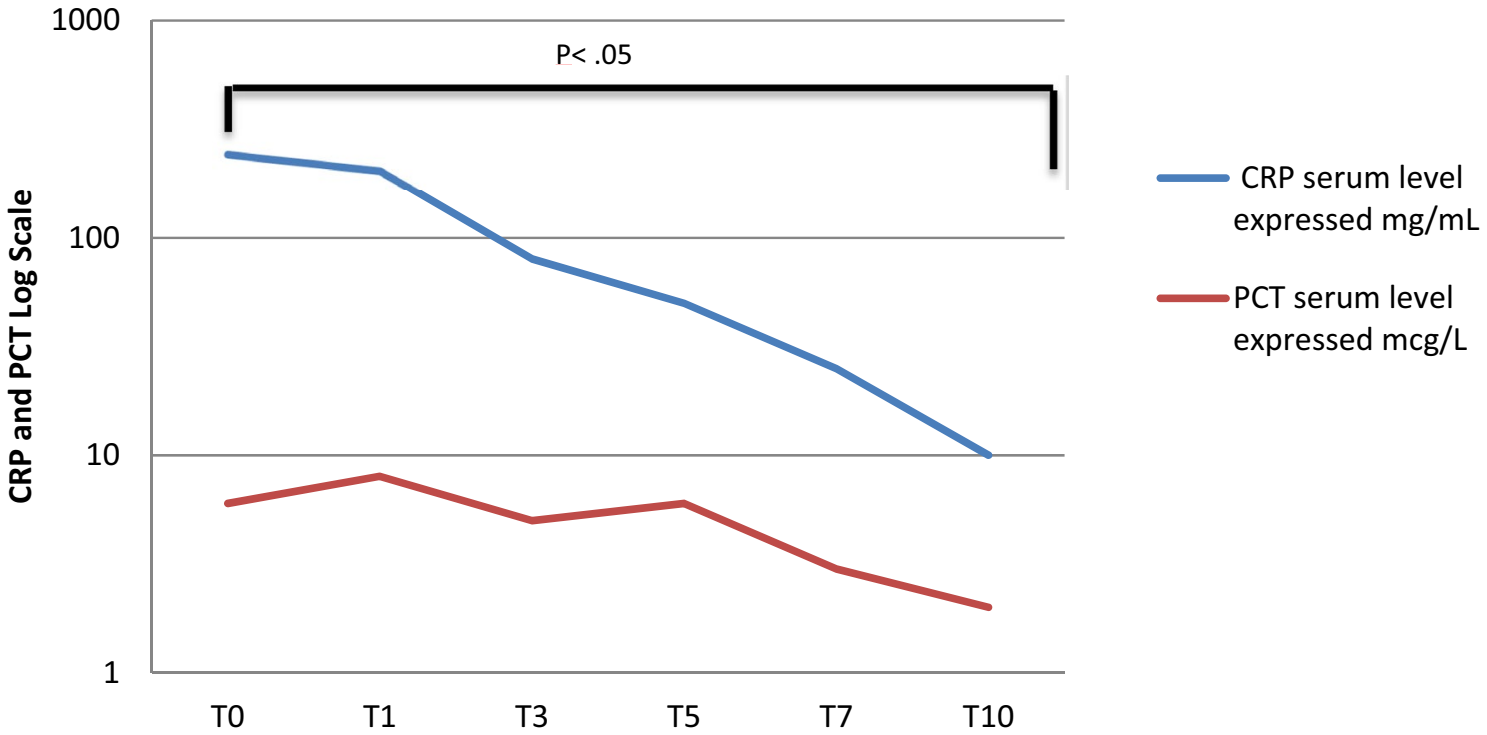

Fig. 1 Figure represents serum levels of CRP and PCT throughout follow-up time (T0, T1, T2, T3 and so on) of the patients expressed as log scale. Data show the reduction of CRP during follow-up in response to antibiotic therapy

had a trend to early increase after surgical procedure in all patients (Tables 1 and 2), (Fig. 1). PCT did not seem to show any difference in serum level following the antibiotic treatment response according to our treatment response criteria ( $U$ Mann-Whitney $p>0.05$ ). Indeed, it was found to be higher in the first $48 \mathrm{~h}$, in those presenting anastomotic dehiscence (PCT 8.4 vs $4.8-U$ Mann-Whitney $p$ 0.004). CRP was increased after surgery in those patients presenting infection nonetheless it showed a statistical significant trend to decrease within $48 \mathrm{~h}$ in those being responsive to antibiotic treatment and with a clinical response within 10 days ( $U$ Mann-Whitney $p=0.01$ ). Further we found that those showing CRP higher than $250 \mathrm{mg} / \mathrm{L}$ had a reduced percentage of success treatment at 14 days compared to those showing CRP $<250 \mathrm{mg} / \mathrm{mL}$ ( $U$ Mann-Whitney $p<0.05$ ). We also managed a multivariate analysis to evaluate possible

Table 3 Mann-Whitney comparison: PCT values at admission (presurgery), first, second, and third day after surgery (T1, T2, and T3) in patients developing postsurgical infection and not

\begin{tabular}{|c|c|c|c|}
\hline & $\begin{array}{l}\text { Patients with postsurgical infections Antibiotic } \\
\text { schedule prior } 2016\end{array}$ & $\begin{array}{l}\text { Patients without postsurgical infections } \\
\text { Antibiotic schedule after } 2016 \text { including } \\
\text { Fosfomycin }\end{array}$ & $\begin{array}{l}\text { Mann- } \\
\text { Whitney } \\
\text { comparison }\end{array}$ \\
\hline & PCT ng/mL, median (IR) & \multicolumn{2}{|l|}{ PCT ng/mL, median (IR) } \\
\hline Presurgery & $0.8(0.6-1.1)$ & $0.75(0.7-1.83)$ & n.s \\
\hline $\mathrm{T} 1$ & $4.6(0.36-6.2)$ & $5.34(0.16-6.92)$ & n.s \\
\hline $\mathrm{T} 2$ & $4.45(0.37-6.1)$ & $3.75(0.15-0.64)$ & n.s \\
\hline \multirow[t]{3}{*}{$\mathrm{T} 3$} & $2.25(0.3-4.6)$ & $1.24(0.12-0.40)$ & $\mathrm{P}=0.001$ \\
\hline & $\begin{array}{l}\text { Patients with postsurgical infections Antibiotic } \\
\text { schedule prior } 2016\end{array}$ & $\begin{array}{l}\text { Patients without postsurgical infections } \\
\text { Antibiotic schedule after } 2016 \text { including } \\
\text { Fosfomycin }\end{array}$ & $\begin{array}{l}\text { Mann- } \\
\text { Whitney } \\
\text { comparison }\end{array}$ \\
\hline & CRP mg/mL, median (IR) & \multicolumn{2}{|l|}{ CRP mg/mL, median (IR) } \\
\hline Presurgery & $178(198-345)$ & $197(174-315)$ & n.s \\
\hline $\mathrm{T} 1$ & $298(210-374)$ & $302(232-394)$ & $\mathrm{P}=0.001$ \\
\hline $\mathrm{T} 2$ & $198(175-225)$ & $135(125-202)$ & $\mathrm{P}=0.0003$ \\
\hline $\mathrm{T} 3$ & $126(107-182)$ & $86(87-132)$ & $\mathrm{P}=0.0001$ \\
\hline
\end{tabular}

This table shows different CRP and PCT levels according to the introduction of Fosfomycin in antibiotic treatment schedule 
influence of different treatment schedule on patients' outcome and the CRP and PCT-related response (Tables 2 and 3). Of note antibiotic treatment started after 2016 and based on the schedule including Fosfomycin showed a faster and better infection outcome compared to those without in terms of Hospital Stay. Mainly they had a rapid improvement of the evaluated parameters for infection and sepsis treatment response, $(36 \mathrm{~h} \pm 6$ vs $48 \mathrm{~h} \pm 12$ ) ( $U$ Mann Whitney $p=0.032$ ) (Table 3). In our cohort of patients we had 27 exitus (15 Group A and 10 in Group B).

\section{Conclusion}

The surgical procedure may be a predisposing factor to infectious complications and consequently sepsis, influencing the patient prognosis, hospital length of stay and therefore being an issue also in antimicrobial resistance onset as well on the cost of patient management $[1,2$, 15]. Therefore, an early diagnosis and prompt treatment may improve the patient's care. Previously some studies have shown that both CRP and PCT levels significantly increase in almost all patients after surgery [3, 4]. Further it has also been found that CRP and PCT levels decrease during antibiotic treatment in patients suffering from VAP (ventilator-associated pneumonia) [5]. On the contrary PCT has been observed to be a good predictor of major anastomotic dehiscence, with a high sensitivity when increase between the third and fifth postoperative days [6]. In our cohort we also found that CRP and not PCT was statistically significant increased in case of infection after surgery and that it has a trend to decrease in response to antibiotic treatment. In fact CRP showed a tendency to decrease in those subjects with antibiotic response and that this response seems to be stronger in those schedule after 2016 including Fosfomycin. Therefore, although CRP has been considered a non-specific inflammation marker, in our cohort seems to be a useful laboratory tool to predict early onset of the infection and antibiotic response, according also to previous evidences too [6]. Particularly it seems that once increased and higher than $250 \mathrm{mg} / \mathrm{dL}$ it seems to be related to the reduced treatment response. Therefore, it is our opinion that a prompt antibiotic treatment CRP driven should be managed as soon as possible once this marker increase after surgical procedures. Further, in the light of the reduction showed in those patients with response to antibiotic treatment, it could be useful to be tested every $48 \mathrm{~h}$, according to our results and protocols. This approach could be significant to assess antibiotic response to promptly change schedule in case of no response. Instead, despite PCT increased in those having non-emergency major abdominal surgery, did not seem to be related to infection onset, antibiotic response or infection resolution in this setting of patients. This finding may be probably due to the stress of surgical procedure or abdominal/hepatic production of cytokines on gut where this molecule is produced too $[8,9]$. Our findings could be of interest because it is the first time, at our knowledge, that CRP and CPT have been tested to evaluate infection after non-emergency major abdominal surgery infection showing interesting results about their usefulness. Multiple events may lead to inflammatory response after major abdominal surgery and most of those are related to massive inflammation following peritoneal damage and organ dysfunction. In this setting of patients underwent elective major abdominal surgery CRP rapidly increases after surgical procedure when an infection is present and it seems to be a useful marker to predict infectious disease evolution, and antibiotic response while PCT seem to be related to surgical procedure complications as previously suggested $[5,10,11,15,16,17]$. It is to underline that our findings are based on data collected in patients undergoing elective surgery. In case of emergency major abdominal surgery different factors may influence the assessed markers as cytokine production following the traumatic stress [18]. In conclusion, it is our opinion that in patients undergoing non-emergency major abdominal surgery CRP and not PCT should be managed to early assess possible infections as well as antibiotic treatment response and that schedule including Fosfomycin may give a clinical advantage in those setting of patients.

Acknowledgements Cardarelli Infectious Disease Study Group is composed: M.Montanari, M. Lanza, I.Guarino, M.Mazzone, A. Basso, M.Perrella, G. Granato, E.Crolla, C. Migliaccio, I.Damiano, M.Grillo, G. Lupone, N.Santomartino, G. Bartone, A. Palumbo.

\section{Declarations}

Conflict of interest The authors declare that they have no conflict of interest.

Research involving human participants and/or animals The study was an observational study according to National, Regional and in Label use of antibiotics.

Informed consent No experimental treatment have been made therefore the patients did not sign any informed consent for any experimental treatment, they just signed informed consent for hospital admission and care.

\section{References}

1. Iwamoto K, Ichiyama S, Shimokata K et al (1993) Postoperative pneumonia in elderly patients: incidence and mortality in comparison with younger patients. Intern Med 32:274-277 
2. Dilworth JP, White RJ (1992) Postoperative chest infection after upper abdominal surgery: an important problem for smokers. Respir Med 86:205-210

3. Angele MK, Faist E (2002) Clinical review: immunodepression in the surgical patient and increased susceptibility to infection. Crit Care 6:298-305

4. Simon L, Gauvin F, Amre DK et al (2004) Serum procalcitonin and C-reactive protein levels as markers of bacterial infection: a systematic review and meta-analysis. Clin Infect Dis 39:206-217

5. Woeste G, Muller C, Bechstein WO, Wullstein C (2010) Increasesedserum levels of C-reactive protein precede anastomotic leakage in colorectal surgery. World J Surg 34:140-146

6. Johannes F, Marcus M, Jochen K et al (2002) Circulating inflammatory and metabolic parameters to predict organ failure after multiple trauma. Eur J Trauma V28:333-339

7. Meisner M, Tschaikowsky K, Hutzler A, Schick C, Schüttler J (1998) Postoperative plasma concentrations of procalcitonin after different types of surgery. Intensive Care Med 24:680-684

8. Lagoutte N, Facy O, Ravoire A, Chalumeau C, Jonval L, Rat P et al (2012) C-reactive protein and procalcitonin for the early detection of anastomotic leakage after elective colorectal surgery: pilot study in 100 patients. J Visc Surg 149:345-349

9. Garcia-Granero A, Frasson M, Flor-Lorente B, Blanco F, Puga R, Carratala A et al (2013) Procalcitonin and C-reactive protein asearly predictors of anastomotic leak in colorectal surgery: a prospective observational study. Dis Colon Rectum 56:475-483

10. McKay GJ, Molloy RG, O'Dwyer PJ (2011) C-reactive protein as apredictor of postoperative infective complications following elective colorectal resection. Colorectal Dis 13:583-587

11. Kiaei BA, Ghiasi F, Moradi D (2015) Precalcitonin and C-reactive protein as markers in response to antibiotic treatment in ventilatorassociated pneumonia in intensive care unit-hospitalized patients. Adv Biomed Res 4:240

12. Horan TC, Andrus M, Dudeck MA (2008) CDC/NHSN surveillance definition of health care-associated infection and criteria for specific types of infections in the acute care setting. Am J Infect Control 36(5):309-332
13. Perrella A, Esposito C, Amato G, Perrella O, Migliaccio C, Pisaniello D, Calise F, Cuomo O, Santaniello W (2016) Antifungal prophylaxis with liposomal amphotericin B and caspofungin in high-risk patients after livertransplantation:impact on fungal infections and immune system. Infect Dis (Lond) 48(2):161-166

14. Eckmann C, Solomkin J (2015) Ceftolozane/tazobactam for the treatment of complicated intra-abdominal infections. Expert Opin Pharmacother 16(2):271-280

15. Sawyer RG, Claridge JA, Nathens AB, Rotstein OD, Duane TM, Evans HL, Cook CH, O'Neill PJ, Mazuski JE, Askari R, Wilson MA, Napolitano LM, Namias N, Miller PR, Dellinger EP, Watson CM, Coimbra R, Dent DL, Lowry SF, Cocanour CS, West MA, Banton KL, Cheadle WG, Lipsett PA, Guidry CA, Popovsky K, STOP-IT Trial Investigators (2015) Trial of short-course antimicrobial therapy for intraabdominal infection. N Engl J Med 372(21):1996-2005

16. Rattan R, Allen CJ, Sawyer RG, Askari R, Banton KL, Claridge JA, Cocanour CS, Coimbra R, Cook CH, Cuschieri J, Dellinger EP, Duane TM, Evans HL, Lipsett PA, Mazuski JE, Miller PR, O'Neill PJ, Rotstein OD, Namias N (2016) Patients with complicated intra-abdominal infection presenting with sepsis do not require longer duration of antimicrobial therapy. J Am Coll Surg 222(4):440-446

17. Jonker MA, Sano Y, Hermsen JL, Lan J, Kudsk KA (2010) Proinflammatory cytokine surge after injury stimulates an airway immunoglobulin a increase. J Trauma 69(4):843-848

18. Perrella A, Esposito C, Pisaniello D, D'Alessio L, Perrella O, Marcos A, Cuomo O (2012) Role of liposomal amphotericin B prophylaxis after liver transplantation compared with fluconazole for high-risk patients. Impact on infections and mortality within one year. Transplant Proc 44(7):1977-1981. https://doi.org/10. 1016/j.transproceed.2012.06.013

Publisher's Note Springer Nature remains neutral with regard to jurisdictional claims in published maps and institutional affiliations. 